\title{
SOUTHEASTERN SECTIONAL SAMPLER
}

In this sampler, the speakers below have kindly provided introductions to their Invited Addresses for the AMS Fall Southeastern Sectional taking place November 3-4 at University of Arkansas, Fayetteville, Arkansas.

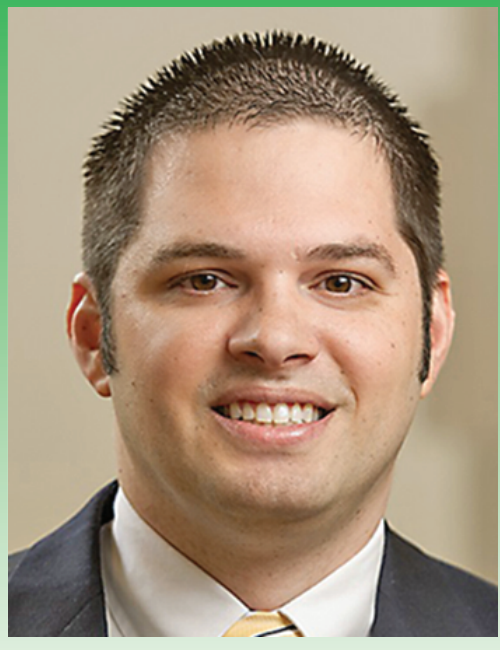

Numerical Algebraic Geometry and Optimization Jonathan D. Hauenstein, University of Notre Dame

page 1251
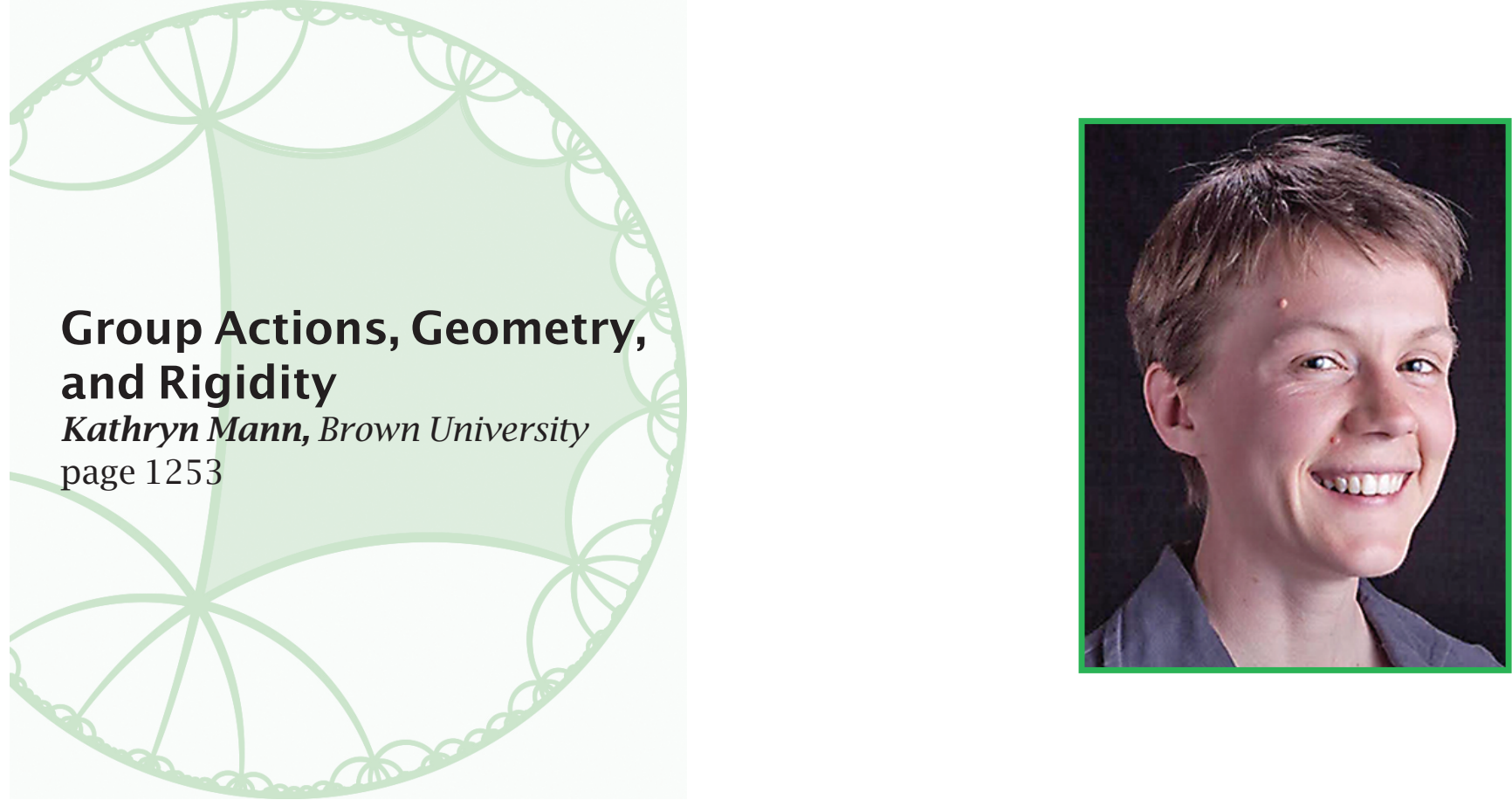

For permission to reprint this article, please contact: reprint-permission@ams.org.

DOI: http://dx.doi.org/10.1090/noti1758 


\section{Jonathan D. Hauenstein}

\section{Numerical Algebraic Geometry and Opti- mization}

Convex programming aims to minimize a convex objective function over a convex set, called the feasible set. For example, linear programming minimizes a linear function over a polytope (intersection of finitely many linear halfspaces as in Figure 1(a)) while semidefinite programming minimizes a linear function over a spectrahedron (intersection of the cone of positive semidefinite matrices with a linear space as in Figure 1(b)).

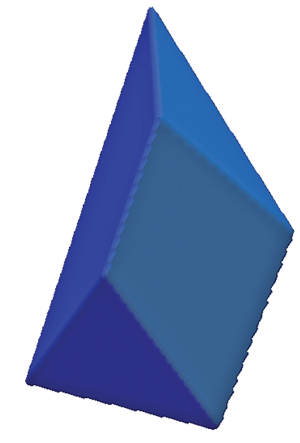

(a)

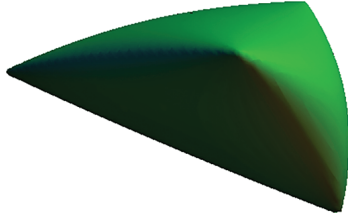

(b)
Figure 1. Example of (a) a polytope and (b) a spectrahedron.

When the feasible set has a nonempty interior, a standard approach for solving convex programs are interior point methods. Conversely, when the feasible set is empty, the program is said to be infeasible and the traditional Farkas' lemma is a standard approach for verifying infeasibility. For example, every infeasible linear program can be verified using the traditional Farkas' lemma. However, there are so-called weakly infeasible semidefinite programs where this is not the case. To illustrate, consider the following semidefinite program:

$\begin{array}{ll}\operatorname{minimize} & x_{11} \\ \text { subject to } & {\left[\begin{array}{cc}x_{11} & 1 \\ 1 & 0\end{array}\right] \succeq 0}\end{array}$

where $A \succeq 0$ means that $A$ is a positive semidefinite matrix. Since the determinant of the matrix in (1) is -1 , the program (1) is clearly infeasible. Moreover, (1) is weakly infeasible since the corresponding alternative via

Jonathan D. Hauenstein is associate professor of applied and computational mathematics and statistics at the University of Notre Dame. His email address is hauenstein@nd.edu.

For permission to reprint this article, please contact:

reprint-permission@ams.org.

DOI: http://dx.doi.org/10.1090/noti1739 the traditional Farkas' lemma is also infeasible, i.e., there does not exist $y \in \mathbb{R}^{2}$ such that

$$
\begin{gathered}
{\left[\begin{array}{cc}
0 & y_{1} \\
y_{1} & y_{2}
\end{array}\right] \geq 0} \\
2 \cdot y_{1}+0 \cdot y_{2}=-1 .
\end{gathered}
$$

One numerical challenge in identifying weakly infeasible semidefinite programs is that perturbations can be strongly infeasible or strictly feasible. For example,

$$
\begin{array}{ll}
\text { minimize } & x_{11} \\
\text { subject to } & {\left[\begin{array}{cc}
x_{11} & 1 \\
1 & \epsilon
\end{array}\right] \succeq 0}
\end{array}
$$

is strongly infeasible for $\epsilon<0$ and strictly feasible for $\epsilon>0$. Liu and Pataki [3] showed that many commonlyused software packages in semidefinite programming have difficulty identifying weakly infeasible semidefinite programs when the reason for infeasibility is not trivially obvious. Such messyinstances were obtained by obscuring their structure via row operations and rotations. Thus, a change of perspective was needed for identifying weakly infeasible semidefinite programs.

Using the lens of numerical algebraic geometry $[1,4]$, the mathematical foundation of traditional interior point methods is to numerically track a solution path of a homotopy from a point in the interior of the feasible set to an optimizer. With this viewpoint, weakly infeasible semidefinite programs can be identified [2] using the following three techniques from numerical algebraic geometry: projective space for compactifying infinite length solution paths, adaptive precision path tracking for navigating through ill-conditioned areas, and endgames for accurately computing singular endpoints.

To illustrate, we consider the following convex program modified from (1):

$$
\begin{array}{ll}
\text { minimize } & \lambda \\
\text { subject to } & {\left[\begin{array}{cc}
x_{11}+\lambda & 1 \\
1 & \lambda
\end{array}\right] \succeq 0 .}
\end{array}
$$

The corresponding optimal value is easily observed to be $\lambda^{*}=0$, but this is actually an infimum that is not attained as a minimum, a condition that is equivalent to (1) being weakly infeasible. Therefore, optimizers to (2) are "at infinity" meaning that a solution path defined by traditional interior point methods will have infinite length and approach an asymptote as represented in Figure 2(a). Compactification using projective space yields a finite length path that can be efficiently tracked as represented in Figure 2(b).

Complex analysis enters the scene to accurately compute the endpoint. The winding number (also called the cycle number) of the endpoint for the path displayed in Figure 2(b) is 2, meaning that the path over the complex numbers locally behaves like the complex square root function. Hence, the Cauchy integral theorem can be used to compute the endpoint of this path by integrating along 


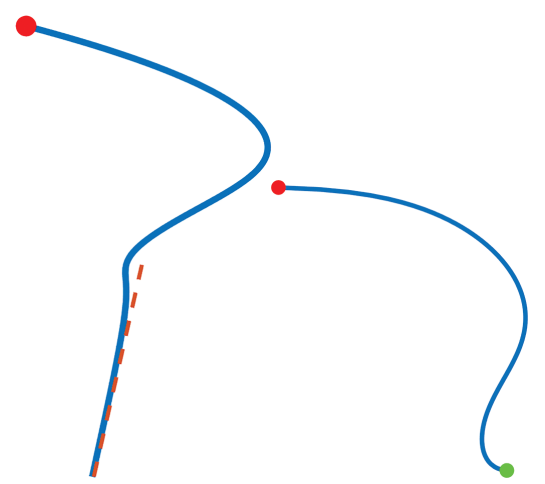

(a)

(b)

Figure 2. (a) A plot of paths at a given (red) point may have infinite length with limiting asymptote corresponding with $\lambda^{*}=0$.

(b) Compactification using projective space yields a finite-length path that can be efficiently tracked.

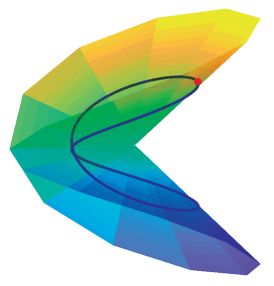

(a)

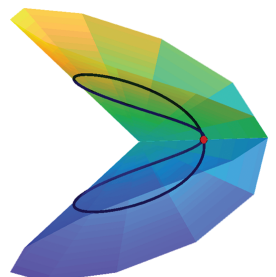

(b)
Figure 3. To compute the endpoint of a path as in Figure 2(b), one uses the Cauchy integral theorem and integrates along a closed loop like the one with winding number 2 with real (a) and imaginary (b) parts pictured here.

a closed loop as shown in Figure 3. Due to periodicity, numerical integration by the trapezoid rule is exponentially convergent [5]. Such a procedure for computing the endpoint is called the Cauchy endgame. Since any endpoint with winding number larger than 1 is necessarily singular, ill-conditioning that necessarily arises near the endpoint can be controlled using adaptive precision path tracking methods.

This viewpoint for identifying weakly infeasible semidefinite programs using numerical algebraic geometry and the software package Bertini [1] along with several other interactions of numerical algebraic geometry and optimization will be discussed in Arkansas.

\section{References}

[1] D. J. Bates, J. D. Hauenstein, A. J. Sommese, and C. W. WAMPLER, Numerically Solving Polynomial Systems with Bertini, volume 25 of Software, Environments, and Tools. Society for Industrial and Applied Mathematics, Philadelphia, PA, 2013. MR3155500

[2] J. D. Hauenstein, A. C. Liddell JR., and Y. ZhanG, Numerical algebraic geometry and semidefinite programming. Preprint available at dx . doi .org/10.7274/R0D798G4.

[3] M. LIU and G. PATAKI, Exact duals and short certificates of infeasibility and weak infeasibility in conic linear programming. Math. Program., 167(2), 435-480, 2018. MR3755739

[4] A. J. SOMmeSE and C. W. WAMPLER, The Numerical Solution of Systems of Polynomials Arising in Engineering and Science. World Scientific, Hackensack, NJ, 2005. MR2160078

[5] L. N. TRefethen and J. A. C. Weideman, The exponentially convergent trapezoidal rule. SIAM Rev., 56(3), 385-458, 2014. MR3245858

\section{Image Credits}

All figures by Jonathan D. Hauenstein.

Photo of Jonathan D. Hauenstein by Matt Cashore, courtesy of the University of Notre Dame.

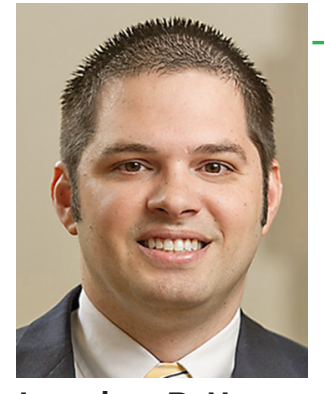

Jonathan D. Hauenstein

\section{ABOUT THE AUTHOR}

Jonathan D. Hauenstein has been honored with a DARPA Young Faculty Award, Sloan Research Fellowship, Army Young Investigator Award, and Office of Naval Research Young Investigator Award. Outside of mathematics, he enjoys spending time with his wife and four daughters. 


\section{Kathryn Mann}

\section{Group Actions, Geometry, and Rigidity}

Classical representation theory is concerned with representations of discrete groups into Lie groups. In topological or smooth dynamics we are concerned with representations of discrete groups into the group of homeomorphisms or diffeomorphisms of a manifold $M$, and the behavior of these representations under perturbation or deformation. Our recent result says that rigidity can arise only in certain geometric ways.

\section{Rigidity}

A representation $\rho$ of a discrete group $\Gamma$ into a topological group $G$ is rigid, loosely speaking, if it has no non-obvious deformations. "Obvious deformations" arise by conjugacy: if $\rho: \Gamma \rightarrow G$ is a representation and $g_{t}$ a path based at the identity in $G$, then $\gamma \mapsto g_{t} \rho(\gamma) g_{t}^{-1}$ gives a continuous path of representations starting at $\rho$. Thus, one way to formalize the notion of rigidity is to define $\rho$ to be rigid if it is an isolated point in the space of representations up to conjugacy, $\operatorname{Hom}(\Gamma, G) / G$.

What is remarkable is that such examples exist at all. Perhaps the most famous rigidity result-and the first theorem that I remember being truly astounded by as a graduate student-is Mostow rigidity. In geometric language, it says that a compact manifold of dimension at least 3 admits at most one hyperbolic structure. In representation-theoretic language, it states that the inclusion $\Gamma \rightarrow \mathrm{SO}(n, 1)$ of a co-compact lattice $\Gamma$ into the Lie group $\mathrm{SO}(n, 1)$, for $n \geq 3$, is rigid in the sense above.

Mostow rigidity completely fails in dimension 2; in fact a genus $g$ compact surface has a much studied $(6 g-6)$-dimensional moduli space of hyperbolic structures. My talk is about how to recover rigidity by passing to the nonlinear, dynamical setting of groups of homeomorphisms.

\section{Geometry and Group Actions}

The story begins with hyperbolic structures on surfaces. If $\Sigma_{g}$ is a surface of genus $g \geq 2$, equipped with a hyperbolic structure, then the universal cover $\tilde{\Sigma}_{g}$ can be identified with the hyperbolic plane and $\pi_{1}\left(\Sigma_{g}\right)$ with a subgroup of the isometry group $\mathrm{SO}(2,1) \cong \operatorname{PSL}(2, \mathbb{R})$. The hyperbolic plane has a natural compactification-in the Poincaré disk model depicted in Figure 1, the compactification adds the circle at the boundary of the open disk-and the action of $\operatorname{PSL}(2, \mathbb{R})$ by hyperbolic isometries of the disc extends to an action on $S^{1}=\mathbb{R} \cup\{\infty\}$ by Möbius transformations. This is an example of what we call a "geometric" action

Kathryn Mann is the Manning Assistant Professor at Brown University. Her email address is mann@math. brown. edu.

For permission to reprint this article, please contact:

reprint-permission@ams.org.

DOI: http://dx.doi.org/10.1090/noti1738

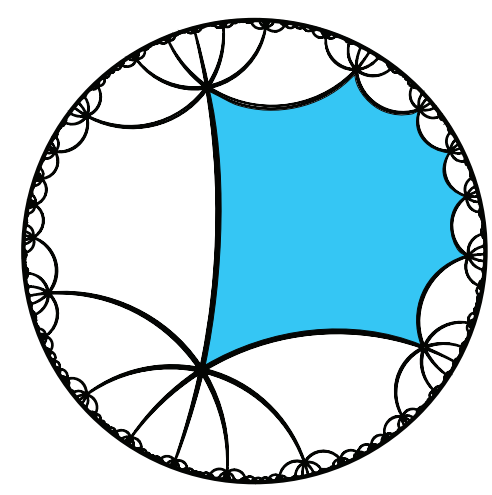

Figure 1. The Poincaré disc model of $\mathbb{M}^{2}$, tiled by fundamental domains for a genus 2 surface. Hyperbolic isometries extend to the boundary and provide an example of a geometric action.

of $\pi_{1}\left(\Sigma_{g}\right)$ on the circle. More generally, we say an action of a discrete group $\Gamma$ on a manifold $M$ is geometric if the action $\Gamma \rightarrow \operatorname{Homeo}(M)$ factors through an embedding $\Gamma \rightarrow G \rightarrow \operatorname{Homeo}(M)$, where $G$ is a connected Lie group acting transitively on $M$, and $\Gamma \subset G$ a co-compact lattice.

It is not difficult to classify all geometric actions of groups on the circle; they are virtually all surface groups, embedded into copies of PSL $(2, \mathbb{R})$ and its central extensions by finite cyclic groups. In earlier work, I showed that these geometric examples were all rigid-they are isolated points in the moduli space of representations of a surface group into $\operatorname{Homeo}\left(S^{1}\right)$. Alternate, independent proofs have since been proposed by S. Matsumoto and J. Bowden.

\section{Hidden Lie Groups}

Recently, Maxime Wolff and I proved the remarkable converse: if $\rho: \pi_{1}\left(\Sigma_{g}\right) \rightarrow \operatorname{Homeo}\left(S^{1}\right)$ is rigid, then $\rho$ is geometric. ${ }^{1}$ In other words, an underlying geometric structure is the only source of dynamical rigidity for surface groups acting by homeomorphisms on the circle.

This result is much more difficult than the original "geometric implies rigid" direction. In that first direction, one is given a geometric representation $\rho$-which can be written down completely explicitly-and one just needs to show that it is stable under perturbation. For the converse, one starts with a completely mysterious representation, save for the knowledge that whatever it is, it can't be deformed. From there, the goal is to conjure up an ambient Lie group.

The proof uses classical dynamical tools such as the rotation number of Poincaré, and various refinements of our own invention, but also mapping class groups and surface topology, a combination theorem for actions admitting Markov-partition-like structures due to Matsumoto, and perspectives borrowed from Calegari, Ghys, and others.

\footnotetext{
${ }^{1}$ Technically, this holds after passing to a Hausdorff quotient of the representation space.
} 
香港中文大學

The Chinese University of Hong Kong

Applications are invited for:-

Department of Mathematics

Professors / Associate Professors / Assistant Professors (Ref. 1800019Z) (Closing date December 31, 2018)

Founded in 1963, The Chinese University of Hong Kong (CUHK) is a forward-looking comprehensive research university with a global vision and a mission to combine tradition with modernity, and to bring together China and the West.

The Department of Mathematics in CUHK has developed a strong reputation in teaching and research. Many faculty members are internationally renowned and are recipients of prestigious awards and honors. The graduates are successful in both academia and industry. The Department is highly ranked internationally. According to the latest rankings, the Department is 29th in the QS World University Rankings and 34th in the US News Rankings.

The Department of Mathematics is now inviting applications for substantiable-track faculty positions at Professor / Associate Professor / Assistant Professor levels. Applicants with strong evidence of outstanding research accomplishments and promise in both research and teaching in Partial Differential Equations, Algebra, Algebraic Geometry, Data Analysis, Geometric Analysis, Mathematical Physics, Probability and Stochastics or related fields are encouraged to apply.

Appointment will normally be made on contract basis for up to three years initially commencing August 2019, which, subject to mutual agreement, may lead to longer-term appointment or substantiation later.

Applications will be considered on a continuing basis but applicants are encouraged to apply by September 30, 2018.

Application Procedure

The University only accepts and considers applications submitted online for the post above. For more information and to apply online, please visit http://career.cuhk.edu.hk.
While the end result has turned into a cohesive narrative, the process felt like a three-year ordeal of "hit it with everything we've got." Fortunately, the philosophy of the proof-constructing geometry from rigidity-can be communicated quite easily in a simplified setting that avoids all the technical nightmare. That's the version you'll see in my talk.

\section{Image Credits}

Figure by Kathryn Mann.

Photo of Kathryn Mann by Jake Paleczny.

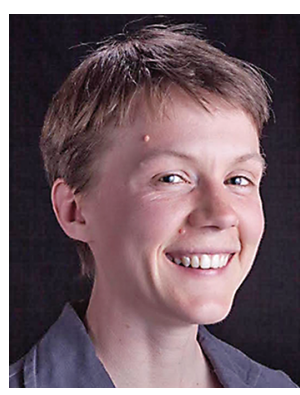

\section{ABOUT THE AUTHOR}

Kathryn Mann works in geometric topology and low-dimensional dynamics, studying moduli spaces of group actions on manifolds. She is the 2019 recipient of the AWMBirman Research Prize in Topology and Geometry. 